\title{
FABRIQUE DES ARCHIVES, FABRIQUE DE L'HISTOIRE
}

\author{
Étienne ANHEIM et Olivier PONCET
}

De récents projets ont posé à nouveau la question de la place des archives et des archivistes dans la société française contemporaine, par rapport aux pouvoirs politiques, à l'opinion publique et aux utilisateurs, à commencer par les chercheurs professionnels ${ }^{1}$. Il est heureux qu'un débat politique se soit instauré publiquement à cette occasion sur ce qui constitue une part du patrimoine national; il nous a paru utile d'entreprendre, en contrepoint, une réflexion collective sur le statut épistémologique des archives et leur place dans la construction du savoir historique. Nous avons voulu construire ce dossier comme un dialogue entre deux professions, les archivistes et les universitaires, et restaurer une distance critique à l'égard des enjeux politicojudiciaires afin de développer une réflexion scientifique autonome sur les archives. Dans ce but, nous voudrions fournir une «situation » du problème, concernant le statut théorique des archives, les rapports pratiques entre les archivistes, les chercheurs et le législateur, enfin, la place du savoir archivistique dans le travail des historiens.

\section{LE STATUT DE L'ARCHIVISTIQUE ET DES ARCHIVES}

Cette réflexion sur le statut des archives et de l'archivistique s'inscrit elle-même dans une tradition déjà ancienne.

En France, la période révolutionnaire, avec son cortège de législations nouvelles, de saisies d'archives privées, de triages et de destructions, constitue le point de départ idéologique et pratique de l'archivistique moderne. Elle inaugura le dernier moment de ce que Robert-Henri Bautier a appelé la «phase cruciale de l'histoire des archives $^{2}$ ». On privilégia tout d'abord les aspects liés au statut des archives - leur publicité et la nationalisation de

1. Voir, p. ex., «Une cité pour les Archives nationales », Les Français et leurs archives. Actes du colloque tenu le 5 novembre 2001 au Conseil économique et social, Paris, Palais d'Iéna, Paris, Fayard, 2002.

2. Bautier, 1968. 
nombreux fonds (ecclésiastiques, d'émigrés) - et les conditions matérielles de leur conservation (institutions, personnels, bâtiments). Puis vint le temps de la mise en ordre. Cette période - qu' ouvre le plan de classement donné par Pierre Daunou aux Archives nationales (1808) et que clôt symboliquement la circulaire du 24 avril 1841 établissant celui des archives départementales, communales et hospitalières - vit s'opérer un basculement des pratiques archivistiques. Le moindre des paradoxes de ce «moment romantique ${ }^{3}$ » ne fut pas la création d'un cadre réglementaire qui faisait peser un danger d'éclatement des fonds de préfectures ou de ministères au moment même où se faisait jour l'adoption comme principe archivistique fondamental de la notion de respect des fonds. Ces contradictions, la célèbre circulaire de 1841 s'efforçait de les dépasser :

«L'expérience a fait reconnaître que le classement ne devait pas être subordonné principalement comme le prescrivait la circulaire du 28 avril 1817 sur ces divisions fondées sur des époques politiques et qu'il faut surtout chercher à les disposer d'après un ordre puisé, non dans le temps mais dans la nature même des documents et l'enchaînement des affaires ${ }^{4}$. »

L'archivistique connut une nouvelle étape décisive sous l'effet du recours plus systématique aux archives pour l'écriture de l'histoire. Les mauristes des $\mathrm{XVII}^{\mathrm{e}}$ et XVIII ${ }^{\mathrm{e}}$ siècles avaient montré le chemin, Michelet en avait tiré un souffle inédit ; il devint le cheval de bataille d'une école positiviste plus diverse qu'on ne l'a dit ${ }^{5}$. La première génération d'archivistes qui n'avaient pas personnellement vécu les événements révolutionnaires accomplit un énorme travail de redécouverte des fonds amassés, dépecés, mutilés avant comme après 1789. Il s'agissait autant de comprendre la logique interne des ensembles documentaires confiés à leur garde que de faciliter leur accès au public par la réalisation d'instruments de recherche ${ }^{6}$ dont les principales formules, encore en vigueur, furent toutes inventées et expérimentées avant 1914. La révolution documentaire de la production écrite de masse toucha les services d'archives dès la seconde moitié du $\mathrm{XIX}^{\mathrm{e}}$ siècle, mais ne provoqua une prise de conscience généralisée qu'au cours des années 1930 et principalement au sortir de la Seconde Guerre mondiale ${ }^{7}$. Une nouvelle archivistique naquit, dominée par le souci d'endiguer le flot de documents, de le canaliser et de le rendre présentable et accessible

3. GAUCHET, 2002.

4. «Instructions pour la mise en ordre et le classement des Archives départementales et communales », Paris, 24 avril 1841, in Lois, instructions et règlements relatifs aux archives départementales, communales et hospitalières [...], Paris, Honoré Champion, 1884, p. 16-28.

5. Hartog, 2001.

6. BIMBENET-PRIVAT, 1991.

7. À titre d'exemple : CELIER, 1950. Il est symptomatique que ce texte, publié dans une revue professionnelle, soit à l'origine une conférence destinée aux élèves sortant de l'École des chartes. 
par de nouveaux instruments de recherche. De ce point de vue, l'irruption informatique et électronique a posé au moins autant de questions qu'elle fournissait de réponses. Dernier avatar de l'archivistique moderne, les archives orales, provoquées, créées par l'archiviste devenu démiurge, cristallisent en elles, à juste titre, l'ensemble des attendus épistémologiques liés aux archives ${ }^{8}$.

Ces textes, que nous avons voulu mettre en évidence ou qui ont pu nous inspirer, montrent que le problème qui se trouve au cœur de toute la réflexion archivistique est celui de la définition même des archives. Il faut rappeler l'importance de la distinction entre archives et documents, fondamentale pour donner au problème posé par l'archivistique toute son ampleur. Les sources archivées dont dispose l'historien ont été fabriquées en deux temps : une première fois en tant que documents, une seconde en tant qu'archives, c'est-à-dire des documents conservés, classés et inventoriés. Or les historiens ont parfois tendance à concentrer leur approche sur une «critique des sources » qui s'intéresse davantage, que ce soit la critique interne ou la critique externe, aux conditions de production et à la signification du texte lui-même, qu'aux conditions de sa conservation et de sa transmission dans des fonds d'archives.

La « mise en archives » des documents est une opération à part entière, qui bouleverse des logiques documentaires pour en créer d'autres, qui affecte à chaque document des coordonnées dans des séries, des fonds, des lieux autant d'éléments qui sont ensuite indissociables du document. L'usage, adopté par certains historiens depuis quelques décennies, du singulier «archive » dénote en ce sens une incompréhension de l'objet historique que constitue un fonds d'archives. Ce singulier revient à réduire le document à une pièce isolée, à faire prévaloir le concept de collection sur celui de fonds d'archives dans lequel le document n'a de sens véritable que rapporté au tout. Les archives ne sont pas de simples témoignages isolés, et la transformation des documents en archives n'a rien de naturel ou d'évident. L'archivage est une opération de construction de catégories, dont l'archivistique doit rendre compte.

Si l'historien ne veut pas être dupe de sa documentation, il est nécessaire qu'il soit conscient de cette double construction, souvent invoquée pour mieux la refouler hors de la conscience professionnelle. En ce sens, l'archivistique doit être considérée comme une discipline historique et non pas seulement comme l'ensemble des techniques de conservation, ou comme une science auxiliaire de l'histoire ${ }^{9}$. L'archivistique, comme « science de la fabrique des archives », peut donner à l'historien les moyens de mieux comprendre comment lui-même « fabrique de l'histoire ».

8. DESCAMPS, 2001.

9. Des journées d'études ont récemment posé la question à des archivistes et à des chercheurs : «L'archivistique est-elle une science ? , Paris, École nationale des chartes, 30-31 janv. 2003. 
Il importe en particulier de savoir distinguer l'usage que le chercheur contemporain peut faire de ses archives - l'usage « historien »- et celui qui en a été fait par les hommes des siècles précédents, qui explique comment et pourquoi ces documents ont été archivés et conservés à travers le temps l'usage « historique ». Cela ne signifie pas qu'il faille limiter le travail de l'historien aux usages historiques de sa documentation, bien au contraire, mais que la compréhension de ces derniers est nécessaire pour éviter les contresens sur les archives. Cette prise en compte de l'archivistique dans le travail de l'historien a d'ailleurs déjà porté ses fruits dans certains domaines de la recherche. Sans insister sur les développements ingénieux auxquels se sont livrés les historiens de l'Antiquité ${ }^{10}$, on peut évoquer la place faite aux archives dans les réflexions sur la culture de l'écrit, que ce soit la question de la mutation graphique et de ses effets, de la literacy ou de l'écriture «pragmatique » (pragmatische Schriftlichkeit) ${ }^{11}$. Les historiens, par un usage plus large de l'archivistique, pourraient sans doute mieux comprendre les limites mais aussi les richesses de leur documentation, en renonçant à la position surplombante qui les pousse parfois à étudier les sources comme si elles avaient été écrites et archivées pour eux.

LES ARCHIVES : DIVERSITÉ DES MÉTIERS, DIFFICULTÉS FACE À LA DEMANDE SOCIALE

Conséquence de cette habitude de pensée, beaucoup d'historiens considèrent que les archives leur sont naturellement destinées, oubliant qu'elles sont au cœur d'une grande diversité de métiers et de demandes sociales. Il faut rappeler que le rapport entre archives et histoire n'est qu' une des facettes de la question posée par les archives à la société. Saisir l'ensemble des difficultés liées aux archives nécessiterait de prendre en compte tous les acteurs qui leur sont liés. Les historiens, «professionnels » en quelque sorte des archives, partagent l'usage des documents avec d'autres publics, historiens dits « amateurs », généalogistes, juristes, journalistes. L'ensemble de ces personnes forme le public des archives et présente des demandes diverses, parfois contradictoires, à un autre corps professionnel, celui des archivistes.

Quelle est la nature de ces demandes ? Si l'on observe les utilisateurs des archives, en laissant de côté les historiens «professionnels » et les personnes qui mènent des recherches de type juridique ou administrative (propriété,

10. FINLEY, 1987.

11. Baldwin, 1991 ; Petrucci, 1993 ; Clanchy, 1993 ; Keller, Meier et Scharff, éd., 1999. En France, le développement de ces problématiques a surtout concerné le Moyen Âge central pour l'instant, voir Guyotjeannin, Morelle et Parisse, 1997. 
filiation, etc.), se dégagent deux grandes orientations, la généalogie et le journalisme, souvent dit «d'investigation » selon la singulière terminologie en cours. La généalogie est emblématique de la pratique quotidienne des salles d'archives, ainsi que d'une époque en proie au «tourment mémoriel », à l'échelle individuelle ou collective. Le journalisme «d'investigation » est le plus souvent représentatif de l'usage positiviste de l'archive, poussé à son extrême : un nom ou un fait, sorti de son contexte, suffisent parfois pour faire un scoop ou une couverture.

Ces deux orientations ne sont pas celle de l'historien; les questions que le journaliste ou le généalogiste posent à leur documentation sont rarement les siennes - en principe... Cependant il arrive qu'il se fasse journaliste, consciemment parfois, mais le plus souvent sans s'en rendre compte : il relaie alors ces interrogations venues du monde des médias ou de la politique pour interroger ses archives, avec toutes les confusions que cela peut entraîner ${ }^{12}$.

De même ces orientations n'ont-elles normalement rien à voir avec le métier d'archiviste. Mais lui aussi épouse parfois les préoccupations de la société qui l'entoure. Certains des discours tenus au moment du débat sur la «Cité des archives ${ }^{13} \gg$ montrent que les archivistes peuvent être eux-mêmes les relais d'une préoccupation mémorielle venue de l'extérieur du champ scientifique même s'il ne faut pas réduire à ce seul aspect une revendication parfaitement légitime quant aux problèmes matériels ou au manque de reconnaissance professionnelle de ce corps par rapport à d'autres, comme ceux des personnels des musées, des monuments historiques ou des bibliothèques. Il est toutefois révélateur que ces revendications aient parfois été défendues par un discours idéaliste, partagé par les universitaires, sur la conservation nécessaire de toutes les archives ${ }^{14}$, appuyé par une rhétorique de la mémoire et du patrimoine qui parfois agit comme « une substance hallucinogène ${ }^{15}$ ».

On mesure dans ce genre d'occasion à quel point les discours extérieurs (par exemple l'interrogation de notre société sur sa mémoire) s'imposent parfois à la pratique professionnelle des chercheurs comme des archivistes. Pourtant, leurs problématiques de réflexion ne peuvent être construites que par une demande externe. La mission de service public assurée par les archivistes, et dans une certaine mesure par les universitaires, ne se résume pas en une adaptation à la « demande de l'opinion publique ». Il importe que les professionnels soient

12. Dumoulin, 2002.

13. La dernière évolution du débat est le communiqué de presse de la présidence de la République, en date du 9 mars 2004, annonçant la décision de lancer la construction d'un nouveau centre pour les Archives nationales à Pierrefitte-sur-Seine (Seine-Saint-Denis), pour une ouverture prévue en 2009.

14. MONNIER, 1999.

15. Pour reprendre l'expression aussi provocatrice qu'heureuse de Michel Melot, voir Melot, 1986. 
conscients de l'existence de cette demande sociale, mais qu'elle soit l'objet d'une analyse, pas d'une simple répétition. C'est aux professionnels qu'il doit revenir d'élaborer collectivement ce qu'ils pensent être les problématiques des rapports entre archives et histoire : nous souhaitons que ce numéro de la Revue de synthèse soit une des occasions de défendre cet espace scientifique autonome, qui se fixe ses propres règles, pour réfléchir ensemble à l'organisation critique du passé qui caractérise l'activité de l'archiviste comme celle de l'universitaire.

Comment situer ces activités l'une par rapport à l'autre ? On échappe mal aux lieux communs. D'un côté, il y aurait une division harmonieuse du travail, aux uns la lecture, l'analyse et la synthèse, aux autres la collecte, l'inventaire et l'indexation. De l'autre, on évoque une opposition dans la démarche : les universitaires influencés par les sciences sociales voudraient penser leur pratique comme la construction d'objets d'étude dans le cadre d'une histoire problème, au contraire, croient-ils, des archivistes, souvent représentés comme les gardiens du patrimoine archivistique, les thésaurisateurs des faits positifs. Ces couples d'opposition sont non seulement faux, mais nuisibles. Passons sur la division du travail, qui produit le plus souvent une hostilité latente entre corps, les universitaires ayant tendance à croire les archivistes à leur service, et ces derniers à considérer les universitaires comme des dangers potentiels pour la conservation de l'intégrité des archives. Passons également sur le fait que beaucoup d'archivistes sont aussi universitaires, et que certains universitaires ont une vraie compétence archivistique ${ }^{16}$. En un sens, la seconde opposition est bien plus fausse. Il n'y a pas, d'une part, la conservation, les faits et les archivistes, de l'autre, la lecture, l'interprétation et les universitaires. Du point de vue du rapport au passé, les deux professions sont situées du même côté, celui d'un travail de critique.

L'archivistique construit les archives et ne se contente pas de les conserver, puisqu'elle les ordonne : une telle activité, avec ce qu'elle fait intervenir de choix et de possibilité, est de l'ordre du retour critique sur le passé. Chaque choix archivistique engage inéluctablement un certain nombre de catégories et d'outils intellectuels qui appartiennent déjà à la pratique historienne, entendue en un sens large. Ainsi, archivistes et universitaires n'ont pas pour tâche

16. Hervé Bastien, futur conservateur à la direction des Archives de France avait ainsi classé et inventorié, en guise de propédeutique, le fonds Henri Queuille conservé au musée Henri-Queuille de Neuvic-Ussel (Archives du président Henri Queuille, conservées au musée Henri-Queuille à Neuvic-Ussel, Paris, Direction des Archives de France, 1989), objet de sa thèse de l'École des chartes (Henri Queuille à Alger ou la République (août 1943août 1944), in Position des thèses [...] de l'École des chartes, Paris, 1989, p. 27-36) ; JeanFrançois Dubost conduisit parallèlement une thèse dédiée aux Italiens en France sous l'Ancien Régime, voir DuBost, 1997, et un guide de recherche plus général, voir DuBOST, 1993 ; enfin, on peut citer un travail réalisé en collaboration par un historien et une archiviste, voir Brian et DEMEUlenaERE-Douyère, dir., 1996. 
l' « antiquariat »; il n'est pas question de conserver le passé tel quel, mais de l'organiser. C'est de cette tâche de mise en ordre du passé que les deux professions doivent être conscientes, pour mieux la satisfaire, et affronter les questions d'une société avide de passé.

En effet, face à l'évolution récente du débat public, il ne paraît pas exagéré de parler de demande sociale à l'égard de la politique des archives, demande plus largement liée à la «conjoncture mémorielle » qui travaille la société française depuis deux ou trois décennies. Le débat est dominé par des problèmes liés aux archives «sensibles » (Vichy, Algérie...) ${ }^{17}$ et aux revendications pratiques. Ces questions ont leur légitimité, mais elles ont tendance à envahir l'ensemble du discours sur les archives, alors qu'elles ne sont pas les seules.

À la double construction de nos sources, comme documents et comme archives, correspond un double problème, politique et épistémologique. Le problème politique, largement illustré dans les débats récents, est celui de l'accès au contenu du document dans des conditions démocratiques. Le problème épistémologique vient de ce que le document est donné à travers une structure archivistique socialement construite, que l'historien doit éclairer et critiquer. Notre choix a été de privilégier le second problème, qui est souvent recouvert par le premier : nous avons essayé de montrer comment les archives posaient un problème scientifique, qu'il nous paraissait important de souligner au moment où la réflexion publique insiste davantage sur le problème politique - comme s'il suffisait de les ouvrir, d'y accéder, pour trouver une vérité qui se donnerait d'un coup au lecteur.

On pourra objecter que tout cela est évident; mais dans quelle mesure le débat actuel prend-il en compte le statut épistémologique des archives ? Rappelons la définition de la loi de 1979 :

«Les archives sont l'ensemble des documents, quels que soient leur date, leur forme ou leur support matériel, produits ou reçus par toute personne physique ou morale, et par tout service ou organisme public ou privé, dans l'exercice de leur activité. »

Cela signifie que tout document appartiendrait aux archives dès le moment où il a été produit, ce qui est contradictoire avec l'idée que les archives sont le produit de deux étapes successives et distinctes, même si pour résoudre, partiellement, cette contradiction, les impératifs dictés par la masse documentaire des archives contemporaines ont conduit à la consécration de la théorie des trois âges des archives (courantes, intermédiaires et définitives ou « historiques »).

Pour le législateur, les archives ne sont pas une construction spécifique, elles se confondent avec les documents, elles sont le devenir naturel de ces documents. Cette naturalisation du statut des archives peut être à l'origine de

17. Un récent colloque vient d'en rendre compte, voir LAURENT, dir., 2003. 
malentendus : en ne séparant pas la production du document de la production des archives, et en naturalisant ces dernières tout en étendant leur définition à l'extrême, la loi crée une confusion, qui pèse sur les débats actuels. Lorsque la loi fait naturellement de tous les documents des archives, elle recouvre les archives par les documents, et du même coup recouvre la question épistémologique - celle de la construction des archives - par la question politique - celle de l'accès au document - qui a tendance alors à occuper tout l'espace. Or si la question politique - comment avoir le plus large accès aux documents ? - est prise pour une question épistémologique, il y a un problème : ce n'est pas dans la conservation la plus large possible et dans l'accès le plus général aux archives que peuvent se résoudre toutes les questions méthodologiques de l'historien, comme cela a été observé à propos de l'ouverture des archives des anciens régimes politiques de l'Europe de l'Est ${ }^{18}$.

Singulièrement, la loi de l'an II était moins oublieuse de la double production des archives - ce qui rappelle au passage qu'avant d'être des objets de l'histoire, les archives sont des objets du droit. Elle reconnaissait clairement que l'archivage était une opération historique, qui transformait le statut des documents auxquels elle s'appliquait. Dès l'article 2, « au corps législatif seul appartient d'ordonner le dépôt aux archives », les archives ne sont pas considérées comme existantes naturellement, virtuellement et de toute éternité : elles sont reconnues comme le produit d'une construction juridique. De même, la loi reconnaît la légitimité des destructions de documents conservés : cette disposition peut choquer aujourd'hui, et on peut regretter la perte d'une documentation parfois extrêmement précieuse, mais il faut reconnaître à la loi le courage de regarder en face le statut des archives, des documents élaborés et réélaborés par le jeu des pratiques sociales et juridiques, et dont l'existence est le fruit d'une construction - sachant qu'il y a forcément une part inéluctable de destruction, puisque non seulement toute la documentation ne peut pas être transformée en archives, mais que rien ne dit qu'elle doive l'être. Cet encadrement des destructions a le mérite d'identifier clairement la question épistémologique, c'est-à-dire la transformation par une société, selon des règles qu'elle se fixe, de documents en archives.

L'évolution postérieure a été un lent et progressif refoulement de cette acceptation initiale du double statut des archives. Peu à peu, décret après décret, la différence entre archives et documents s'est estompée, jusqu'à ce que s'impose l'idée que de droit, par nature, tout document produit est dès son origine une pièce d'archives. Le célèbre décret de Jean Zay en date du 21 juillet 1936, consacré aux versements dans les dépôts d'archives d'État des papiers des ministères et des administrations qui en dépendent, marque dans cette évolution une inflexion notable dans la définition des archives : « Les

18. FrançoIs, 1995 ; Wolikow, 1997. 
dossiers, registres et pièces reconnus inutiles pour les ministères, administrations, service et établissements de l'État. » On remarque le paradoxe : alors qu'au $\mathrm{XIV}^{\mathrm{e}}$ siècle, les archives sont définies comme les documents qu'il faut garder car ils sont utiles à l'État, elles deviennent les documents qui n'ont plus d'utilité pour lui. Pointe derrière cette évolution la montée en puissance du discours historien sur les archives : elles n'ont plus d'utilité pour l'État mais c'est alors qu'elles en ont pour nous. La loi de 1979 représente une victoire pour cette conception extensive des archives, mais peut-être y a-t-il aussi là un danger.

Il ne faut certes pas négliger le vécu de la loi : derrière des propositions maximalistes, la pratique est marquée par une jurisprudence qui va dans le sens d'une destruction réfléchie d'une partie de la documentation - n'a-t-on pas écrit que l'archiviste « est devenu en quelque sorte le spécialiste de l'élimination: il est l'homme qui sait détruire $^{19} »$ ? La direction des Archives de France élabore ainsi, depuis plusieurs décennies et sous des dénominations diverses, des tableaux de gestion pour guider la politique de tri.

Reste toutefois que le point de vue du législateur, présent dans le texte de l'an II, assumant son rôle d'instance de transformation des documents en archives, semble remplacé dans la loi de 1979 par le point de vue, au moins théorique, de l'historien. Pour lui, tout document archivé peut devenir une source, de sorte qu'il peut rêver de la conservation la plus large possible. Cette ambition est bien compréhensible, mais doit-elle se substituer à la volonté du législateur? C'est ce qui se passe quand tout est défini comme archives, sans égard pour la nécessaire transformation du document en pièce d'archives. En adoptant le point de vue de l'historien, la loi de 1979 universalise une position spécifique, celle du professionnel de l'analyse historique, qui prend le pas sur l'archivistique - cette dernière étant condamnée à suivre un groupe de professionnels, lui-même soumis à la pression d'une conjoncture mémorielle, pour aboutir à une définition des archives qui conduirait dans la pratique à faire des archivistes autant de Danaïdes.

Ces préoccupations visant à mettre en évidence le problème épistémologique posé par les archives, souvent dissimulé par le problème politique, nous ont conduits à minorer la place accordée à l'histoire contemporaine dans notre travail collectif. Il ne s'agit pas d'un mépris ou d'une ignorance : l'attitude critique qui nous a inspirés est illustrée par de nombreux travaux d'historiens du $\mathrm{XX}^{\mathrm{e}}$ siècle, comme le montre la réfutation du néopositivisme par Jean-Marc Berlière ${ }^{20}$. C'est un choix raisonné de rééquilibrage : il nous a paru intéressant de donner la parole à des chercheurs travaillant sur des archives

19. BAUTIER, 1961, p. 1161.

20. BERLIÈRE, 2001. 
plus anciennes, moins sujettes à la polémique extra-scientifique, pour essayer d'illustrer notre approche des rapports entre archivistique et historiographie, mais aussi pour dialoguer avec la bibliographie déjà existante, nettement dominée par les historiens du monde contemporain.

\section{ARCHIVISTIQUE ET ÉCRITURE DE L'HISTOIRE}

Nous avons voulu souligner dans ce dossier la dépendance de l'historien à l'égard non seulement de sa documentation, mais de la manière dont celle-ci a été conservée. Les archives représentent une forme de détermination pour le travail de l'historien. Détermination absolue d'abord : si l'on exclut « l'histoire du temps présent », la masse des archives, si énorme soit-elle, représente un corpus clos, dont les lacunes ne peuvent être comblées que marginalement (sauf dans le cas de la constitution d'archives orales) par de fortuites découvertes, mais qui reste un facteur limitatif de n'importe quelle enquête historique. Certaines enquêtes sur des périodes données sont simplement impossibles. Mais la détermination est aussi relative, non pas du point de vue de l'absence de documentation, mais de la manière dont celle-ci se présente. Les formes de classement des archives, qui sont au départ des catégories instituées - ce qui ne signifie pas sans raison - finissent par constituer un cadre naturel pour le chercheur et l'archiviste qui les emploient au quotidien. Ces frontières et ces catégories ont un retentissement sur la pratique même des historiens, qui n'en sont pas toujours conscients.

Le problème se pose de manière particulièrement cruciale dans les travaux qui concernent plusieurs États, tant il est vrai que le classement des archives est aussi affaire de traditions nationales, et que l'organisation de la documentation n'est pas du tout la même en Italie, en France ou en Allemagne, par exemple même si aujourd'hui les organismes internationaux de coopération tentent de donner, pour les archives contemporaines, des définitions communes et des normes internationales.

La mise en évidence de ces limites ne signifie pas qu'une forme d'objectivité historique soit impossible à atteindre. L'archivistique ne doit pas pousser à conclure à la relativité absolue de toute construction intellectuelle élaborée à partir des archives - et donc à l'inutilité du travail de dépouillement et d'érudition. L'étude des archives peut, entre positivisme et relativisme, conduire l'historien à un rationalisme critique dans son travail : exhiber les catégories inconscientes à l'œuvre dans le classement des archives est sans doute la meilleure manière de s'en défaire.

Dans cette perspective, notre dossier est construit autour de deux axes complémentaires. Le premier concerne l'histoire des archives et de leur traitement 
archivistique, et vise à illustrer le fait qu'elles sont des produits socioculturels complexes. C'est ainsi qu'Olivier Guyotjeannin et Yann Potin montrent à partir de l'exemple du « Trésor des chartes » comment histoire et archivistique peuvent se conjuguer : étudier la constitution d'un dépôt dans le temps long, du XIII ${ }^{\mathrm{e}}$ siècle à la fin de l'Ancien Régime, permet d'expliquer la place de cet ensemble dans l'historiographie contemporaine. C'est une autre grande série des Archives nationales, celle des archives du parlement de Paris, qui est l'objet de l'analyse de Françoise Hildesheimer. Rappelant que les archives ne sont pas seulement des textes, mais le produit de pratiques dont il faut restituer la logique, elle montre comment l'histoire du fonds est un préalable nécessaire à son exploitation. Plus encore, elle met en évidence le rôle du traitement archivistique, par exemple avec la politique d'inventaires, dans l'utilisation de ces archives par les historiens, de sorte qu'elle propose finalement de doubler l'histoire du fonds par une histoire de l'archivistique contemporaine de ce fonds, manière de soustraire l'historien aux pesanteurs invisibles de sa documentation.

Le second axe, complémentaire, consiste à se placer non plus du point de vue des fonds, mais de celui des objets étudiés par les historiens, pour tenter de voir comment ils peuvent être affectés par une approche archivistique. C'est à partir d'exemples tirés de l'histoire de la parenté - occasion de rappeler, contrairement aux idées reçues, que l'histoire sociale est toujours capable de formuler des propositions épistémologiques nouvelles - que Joseph Morsel et François-Joseph Ruggiu ont illustré ce projet. En posant le problème du concept de « lignage » à propos de l'aristocratie allemande à la fin du Moyen Âge, Morsel montre les effets de réel qu'a pu produire sur des générations d'historiens une structure archivistique qui fut précisément constituée par certaines familles, afin d'accréditer l'existence ancienne de ces prétendus « lignages ». Comme il l'écrit,

« des aristocrates des siècles antérieurs sont rétrospectivement transformés en ancêtres, et c'est en ancêtres qu'on tend à les étudier, important ainsi, à partir de ces fonds, la logique institutionnelle du Geschlecht dans des temps où ils n'ont que faire ».

Ruggiu, pour sa part, étudie la famille au XVIII ${ }^{\mathrm{e}}$ siècle en France et en Angleterre et pose de manière comparatiste le problème des effets historiographiques de la structure archivistique. Les deux sociétés sont-elles réellement très différentes ou les différences observées ne viennent-elles pas essentiellement de la nature des sources conservées ? Cette question a un corollaire : deux sociétés différentes par nature ne produisent-elles pas de toute façon des sources différentes?

Enfin, nous avons voulu compléter ce dossier avec deux autres contributions. L'une consiste en une réflexion critique sur la notion d' "archive », telle 
qu'elle est employée dans le dernier ouvrage de Paul Ricœur, et sur l'usage que les historiens peuvent en faire face à leurs archives. L'autre est une bibliographie raisonnée, qui pourra aider le lecteur à trouver son chemin dans les nombreuses publications sur les archives et leur histoire.

Une meilleure prise en compte de l'archivistique constituerait, sans doute, une manière de renouveler les approches historiques, non seulement pour l'époque contemporaine mais également pour les périodes plus anciennes. L'évolution historiographique de la décennie écoulée, l'actualité de la recherche ${ }^{21}$ comme celle de l'édition montrent à quel point nos propos, loin d'être incantatoires, s'inscrivent au contraire dans des préoccupations partagées par une partie de la communauté des historiens - à condition de bien distinguer démarche politique et démarche épistémologique dans le domaine des archives, la seconde pouvant contribuer à un regard, sinon apaisé, du moins plus scientifique sur la première. Face au fantasme mémoriel, la tâche pour les historiens comme pour les archivistes n'est pas de conserver au mieux, et en plus grande quantité, ce passé sous forme de mémoire brute, mais de l'ordonner, de le critiquer, en un sens de le liquider, d'en débarrasser la vie sociale en l'organisant. Les archives posent le problème de l'organisation de notre rapport au passé et à notre mémoire : il s'agit de construire une histoire pour éviter qu'elles ne soient un fardeau, au sens figuré, mais aussi au sens propre, si l'on en juge par l'abondance de la documentation à conserver.

Étienne ANHEIM

École française de Rome
Olivier PONCET

CHAN-École nationale des chartes

(mars 2004).

21. En témoignent, p. ex., les séminaires consacrés aux archives à l'École des hautes études en sciences sociales : «Archives et sciences sociales », sous la responsabilité d'Éric Brian, Jean Jamin, Morgane Labbé, Pap Ndiaye et Vincent Duclert ; « Histoire de la documentation historique », sous la responsabilité de Vincent Duclert, Anita Guerreau-Jalabert et Françoise Hildesheimer. 


\section{LISTE DES RÉFÉRENCES}

BAldwin (John), 1991, Philippe Auguste et son gouvernement. Les fondations du

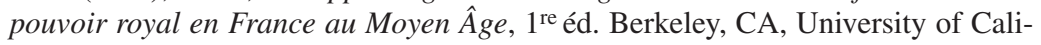
fornia Press, 1986, ici Paris, Fayard.

BAUTIER (Robert-Henri), 1961, «Les archives », in SAMARAN (Charles), dir., L'Histoire et ses méthodes, Paris, Gallimard (Encyclopédie de la Pléiade, 11), p. 1120-1167.

BAUTIER (R.-H.), 1968, « La phase cruciale de l'histoire des archives. La constitution des dépôts d'archives et la naissance de l'archivistique, XVI ${ }^{\mathrm{e}-d e ́ b u t ~ d u ~ X I X ~}{ }^{\mathrm{e}}$ siècle », Archivum, 18, p. 139-150.

BERLIÈre (Jean-Marc), 2001 « Archives de police/historiens policés ? », communication à la table ronde «Les historiens et les archives » de la Société d'histoire moderne et contemporaine, Paris, 31 mars 2001, Revue d'histoire moderne et contemporaine, t. XLVIII, 4bis, suppl., p. 57-68.

BIMBENET-PRIVAT (Michèle), 1991, «Les répertoires numériques rédigés aux Archives nationales vers 1890. Le travail d'une génération », La Gazette des archives, 152-153, p. 48-54.

BriAn (Éric) et DEMEUlENAERE-DouYÈre (Christiane), dir., 1996, Histoire et mémoire de l'Académie des sciences. Guide de recherches, Paris-New York, Technique \& documentation Lavoisier.

CELIER (Léonce), 1950, «La responsabilité de l'archiviste dans l'élimination des papiers inutiles », La Gazette des archives, 7, p. 9-14.

Clanchy (Michael), 1993, From memory to written record. England 1066-1307, $1^{\text {re }}$ éd. Londres, Edward Arnold, 1979, ici $2^{\mathrm{e}}$ éd. augm., Oxford, UK-Cambridge, MA, Blackwell.

DesCAMPS (Florence), 2001, L'Historien, l'archiviste, le magnétophone. De la constitution de la source orale à son exploitation, Paris, Comité pour l'histoire économique et financière de la France (Histoire économique et financière de la France, Sources).

Dubost (Jean-François), 1993, Les Étrangers en France, XVI ${ }^{e}$ siècle-1789. Guide des recherches aux Archives nationales, Paris, Archives nationales.

Dubost (J.-F.), 1997, La France italienne, XVI ${ }^{e-X V I I^{e}}$ siècle, Paris, Aubier (Aubier histoires).

Dumoulin (Olivier), 2002, Le Rôle social de l'historien. De la chaire au prétoire, Paris, Albin Michel (Bibliothèque histoire).

FINLEY (Moses I.), 1987, « Le document », in ID., Sur l'histoire ancienne. La matière, la forme et la méthode, $1^{\mathrm{re}}$ éd. " Documents », in ID., Ancient History. Evidence and models, Londres, Chatto and Windus, 1985, p. 27-46, ici Paris, La Découverte (Textes à l'appui. Histoire classique), p. 68-96.

FRANÇOIS (Étienne), 1995, «Les "trésors" de la Stasi ou le miroir des archives », Autrement, 150-151, janv., p. 145-151. 
GaUCHET (Marcel), 2002, dir., Philosophie des sciences historiques. Le moment romantique. Textes de P. Barante, V. Cousin, F. Guizot, J. Michelet, F. Mignet, E. Quinet, A. Thierry, $1^{\text {re }}$ éd. Lille, Presses universitaires de Lille, 1988, ici repr. Paris, Seuil (L'histoire en débats. Points Histoire).

Guyotjeannin (Olivier), Morelle (Laurent) et Parisse (Michel), 1997, « Pratiques de l'écrit documentaire au $\mathrm{XI}^{\mathrm{e}}$ siècle », $\mathrm{n}^{\circ}$ spéc., Bibliothèque de l'École des chartes, t. CLV, fasc. 1, p. 7-339.

Hartog (François), 2001, Le XIX Siècle et l'histoire. Le cas Fustel de Coulanges, $1^{\text {re }}$ éd. Paris, Presses universitaires de France, 1988, ici réimpr. Paris, Seuil (Points Histoire).

Keller (Hagen), MeIer (Christel) et ScharfF (Thomas), éd., 1999, Schriftlichkeit und Lebenpraxis im Mittelalter. Erfassen, Bewahren, Verändern, Munich, W. Fink.

LAURENT (Sébastien), dir., 2003, Archives « secrètes », secrets d'archives ? Historiens et archivistes face aux archives sensibles, Paris, CNRS Éditions (CNRS Histoire).

Melot (Michel), 1986, « Des archives considérées comme substance hallucinogène », Traverses, 36, p. 14-19.

Monnier (François), 1999, «De l'intérêt historique. À propos d'un avant-projet de loi sur les archives », Revue administrative, 309, p. 227-229.

PetruCCI (Armando), 1993, Jeux de lettres. Formes et usages de l'inscription en Italie (XI ${ }^{e-} X^{e}$ siècle), Paris, Éditions de l'École des hautes études en sciences sociales.

Wolikow (Serge), 1997, Une histoire en révolution? Du bon usage des archives de Moscou et d'ailleurs, Dijon, Éditions universitaires de Dijon. 